\title{
Production of hadron exotics in high energy processes
}

\author{
Wei Wang ${ }^{1, \star}$ \\ ${ }^{1}$ INPAC, Shanghai Key Laboratory for Particle Physics and Cosmology, \\ Department of Physics and Astronomy, Shanghai Jiao-Tong University, Shanghai 200240, China
}

\begin{abstract}
Production of hadron exotics in high energy processes may play a role in unravelling their nature. In high energy limit, the leading power behavior of the cross section of exclusive processes is usually encoded in the constituent counting rule. In this talk I will demonstrate for the process involving hadrons with hidden flavors the constituent counting rule requests modifications. I will use effective field theory to derive the power scalings. After the $e^{+} e^{-} \rightarrow V P$ example I will discuss the production of hadron exotics including the $X(3872)$ and $Z_{c}^{ \pm}$in high energy process. I will also briefly talk about the challenge of the molecular interpretation of $X(3872)$ based on its large production rates in hadron collisions.
\end{abstract}

\section{Introduction}

Thanks to worldwide experiments during the last decades at $e^{+} e^{-}$and hadron colliders, our interest in hadron spectroscopy has been revived by the discovery of a plethora of new hadrons. Most of them were discovered in the charmonium and bottomonium sector and many defy a standard quarkonium interpretation. A recent review can be found in Ref. [1].

\section{Constituent scaling rule for hadron exotics in exclusive production}

\subsection{Constituent scaling rule}

At high energy with $\sqrt{s} \gg \Lambda_{\mathrm{QCD}}$ where $s$ is the center-of-mass energy squared and $\Lambda_{\mathrm{QCD}}$ is the confinement scale, exclusive processes can be understood in QCD perturbation theory. The leading power behavior will lead to a constituent scaling rule in the factorization limit and can be formally derived by matching the full theory of QCD to a low-energy effective field theory (EFT).

At high energy, the differential cross-section of a generic process $a+b \rightarrow c+d$ obeys the asymptotic behavior [2]:

$$
\frac{d \sigma}{d t} \sim s^{2-n} f\left(\theta_{c m}\right)
$$

with $n=n_{a}+n_{b}+n_{c}+n_{d}$, and $a, b, c, d$ denoting generic leptons, photons or hadrons. In the above, the $s$ and $t$ are Mandelstam variables, and the $\theta_{c m}$ is the scattering angle in the center-of-mass frame.

^e-mail: wei.wang@sjtu.edu.cn 
Here $n_{h}$ is the number of constituents in the particle $h$. A fundamental particle with no substructure like a quark, electron, or a photon has $n_{i}=1$. An ordinary meson has $n_{i}=2$ and a baryon has $n_{i}=3$.

Recently, it has been argued in Refs. [3-7] that the cross section for high energy production of multiquark states should also scale as Eq. (1) with the power of $s$ determined according to their expected valence quark structures. Instead of $n_{i}=2$ for an ordinary meson, a meson-meson molecule or a compact tetraquark has $n_{i}=4$, and a pentaquark has $n_{i}=5$.

If the constituent counting rules were correct, it would provide a very straightforward tool to unravel the quark structures of exotic hadrons. In the following, I will show for hadrons with hiddenflavor quarks it is problematic to naively apply such a constituent counting rule [8]. I will consider a simple example involving only ordinary mesons, $e^{+} e^{-} \rightarrow V P$, with $V$ and $P$ denoting ordinary light flavor vector and pseudoscalar mesons, respectively. This process requests a helicity flip and thus it implies the following scaling for integrated cross section [2] from Eq. (1):

$$
\sigma\left(e^{+} e^{-} \rightarrow V P\right) \propto \frac{1}{s^{4}}
$$

\subsection{SCET power counting}

Perturbation theory at high energies allows to express the matrix elements of Heisenberg operators in terms of free local operators and interaction terms. When factorization exists, one may separate the interactions according to the involved scales using the operator product expansion. For a generic matrix element, we have:

$$
\begin{aligned}
\left\langle f\left|O_{H}(0)\right| i\right\rangle & =\left\langle f\left|T\left[O \times \exp \left[i \int d^{4} x \mathcal{L}_{\text {int }}(x)\right]_{>\mu} \times T \exp \left[i \int d^{4} x \mathcal{L}_{\text {int }}(x)\right]_{<\mu}\right]\right| i\right\rangle \\
& \sim\left\langle f\left|T\left[O^{\prime} \times \exp \left[i \int d^{4} x \mathcal{L}_{\text {int }}(x)\right]_{<\mu}\right]\right| i\right\rangle \equiv\left\langle f\left|O_{H, \mu}^{\prime}\right| i\right\rangle,
\end{aligned}
$$

where we have formally integrated out the interactions above the factorization scale $\mu$, and obtained a new set of low-energy effective operators $O^{\prime}$. The interaction below $\mu$ contains no information on the $1 / \sqrt{s}$ and thus the scaling is obtained by counting the pertinent number of constituents in $O^{\prime}$.

Now I will use the effective field theory to explicitly derive the leading power contribution to the cross section. The applicable EFT here is the soft-collinear effective theory (SCET) [9, 10]. For an introduction please see the textbook [11]. Instead of directly studying the $s$ dependence, we introduce a dimensionless parameter $\lambda=\Lambda / \sqrt{s}$ and and count the power dependence on $\lambda$

$$
\sigma \sim \frac{1}{s}\left(\frac{\Lambda}{\sqrt{s}}\right)^{n} .
$$

The $\Lambda$ is a low-energy scale and may be taken as $\Lambda_{Q C D}$ in the case of a light quark, $m_{c / b}$ in the case of a charm/bottom quark.

At high energy, the energetic quarks are jet-like (collinear) with the typical momentum

$$
p=\left(p^{+}, p^{-}, p_{\perp}\right) \propto\left(\sqrt{s}, \frac{\Lambda^{2}}{\sqrt{s}}, \Lambda\right) .
$$

For an energetic quark, it is convenient to split the quark field into two components:

$$
\psi=\xi+\eta, \quad \xi=\frac{\not h \hbar}{4} \psi, \quad \eta=\frac{\not h h}{4} \psi,
$$




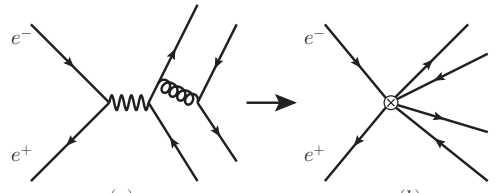

(a)

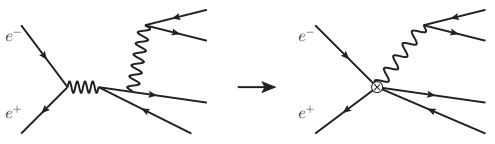

$(b)$
Figure 1. Feynman diagram for $e^{+} e^{-} \rightarrow V P$ in the full theory (a) and effective field theory (b).

Figure 2. Feynman diagram for the photon contribution to $e^{+} e^{-} \rightarrow V P$ in the full theory (a) and effective field theory (b). Here the vector meson is a neutral one like $\rho^{0}, \omega, \phi$.

where $n$ and $\bar{n}$ is two light-like vectors: $n^{2}=\bar{n}^{2}=0$. The quark field scaling can be obtained by considering the two-point correlator:

$$
\langle 0|T[\psi(x) \bar{\psi}(y)]| 0\rangle=\int \frac{d^{4} p}{(2 \pi)^{4}} e^{-i p \cdot(x-y)} \frac{i(\not p+m)}{p^{2}-m^{2}+i \epsilon} .
$$

This gives $\xi \propto \lambda, \eta \propto \lambda^{2}$. For a collinear photon or gluon field, one similarly finds $n_{+} A \propto 1, \quad A_{\perp} \propto$ $\lambda, \quad n_{-} A \propto \lambda^{2}$. From the normalization, the mesonic state scales as $|M\rangle \propto \lambda^{-1}$.

Matching to the effective field theory is equivalent to integrate out the off-shell intermediate states. For the $e^{+} e^{-} \rightarrow V P$ shown in Fig. 1, the vector meson is produced by a pair of quark fields with:

$$
\left\langle V\left|\bar{\psi} \gamma_{\perp}^{\mu} \psi\right| 0\right\rangle=\left\langle V\left|\left(\bar{\xi} \gamma_{\perp}^{\mu} \eta+\bar{\eta} \gamma_{\perp}^{\mu} \xi\right)\right| 0\right\rangle \sim \lambda^{2},
$$

where the transverse projection receives the additional suppression $\lambda$. The leptonic state cancels the scaling of the lepton field. Then the power scaling of amplitude for $e^{+} e^{-} \rightarrow V P$ is given as

$$
\mathcal{M} \propto\langle P|\bar{\xi} \hbar \xi| 0\rangle \times\left\langle V\left|\bar{\psi} \gamma_{\perp}^{\mu} \psi\right| 0\right\rangle \propto \lambda^{3},
$$

and the cross section has

$$
\sigma \propto \frac{1}{s} \lambda^{6} \propto \frac{\Lambda^{6}}{s^{4}}
$$

This recovers the standard perturbative QCD prediction given in Eq. (2).

A vector meson such as $\rho^{0}$ can be produced by a photon field $\left\langle\rho^{0}\left|A_{\perp}^{\mu}\right| 0\right\rangle$. A gauge invariant operator can be constructed by including the Wilson line. Then the scaling of decay amplitude

$$
\mathcal{M} \propto\langle\pi|\bar{\xi} \hbar \xi| 0\rangle \times\left\langle\rho^{0}\left|A_{\perp}^{\mu}\right| 0\right\rangle \propto \lambda,
$$

leads to the cross section

$$
\sigma \propto \frac{1}{s} \lambda^{2} \propto \frac{\Lambda^{2}}{s^{2}} .
$$

It is interesting to notice the production through a photon is suppressed by the electromagnetic coupling constant $\alpha_{\mathrm{em}} \sim 1 / 137$ and less important at low energies. But at very high energies this new diagram will dominate and in particular it gives a scaling rule different from the one in Eq. (2) [12].

To understand the above behaviors and accommodate with the constituent scaling rule, one can technically count the valence degrees of freedom of the neutral vector meson as $n_{i}=1$, which amounts to count the number of lines (a photon in this case) attached to the effective vertex. The lesson that one can learn from the above example is: not all ingredients undergo the hard momentum transfer. The fall-off scaling is determined by the leading-power (leading-twist) operator at the scale $\mu=\sqrt{s}$ which overlaps with the hadron. 


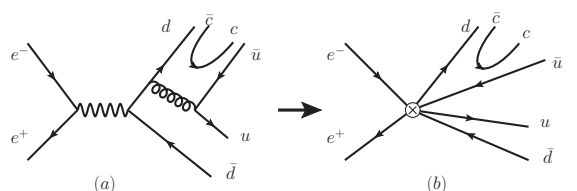

Figure 3. Feynman diagrams for $e^{+} e^{-} \rightarrow Z_{c}^{ \pm} \pi^{\mp}$.

\subsection{Implications}

Let us switch to the exclusive production of multiquark states, and consider the $e^{+} e^{-} \rightarrow Z_{c}^{ \pm} \pi^{\mp}$. The $Z_{c}^{ \pm}(3900)$ was discovered in Refs. [13, 14], but its nature is still controversial. In Ref. [6], it has been argued that its cross section in the $s \rightarrow \infty$ limit obeys the scaling

$$
\frac{\sigma\left(e^{+} e^{-} \rightarrow Z_{c}^{+}(\bar{c} c \bar{d} u) \pi^{-}(\bar{u} d)\right)}{\sigma\left(e^{+} e^{-} \rightarrow \mu^{+} \mu^{-}\right)} \stackrel{?}{\propto} \frac{1}{s^{4}},
$$

where the $Z_{c}^{+}(\bar{c} c \bar{d} u)$ is a tetraquark made of two quarks and two antiquarks. We have put a question mark to the above scaling behavior since we believe that it is problematic at very high energies. We show a production mechanism in Fig. 3 (a). In this diagram, the heavy quark pair $\bar{c} c$ is generated from the QCD vacuum, and such a contribution is suppressed by $O\left(1 / \mathrm{m}_{c}^{2}\right)$. Integrating out the off-shell intermediate propagators at the scale $\sqrt{s}$ we find that the $Z_{c}$ behaves as an ordinary $\bar{q} q$ meson and the $s$ dependence scaling of the cross-section is determined by light quarks of the $Z_{c}$ :

$$
\frac{\sigma\left(e^{+} e^{-} \rightarrow Z_{c}^{+} \pi^{-}\right)}{\sigma\left(e^{+} e^{-} \rightarrow \mu^{+} \mu^{-}\right)} \propto \frac{1}{s^{2}},
$$

which can be derived by counting the number of lines attached to the effective vertex. It is obvious this production mechanism will become dominant at very high energies with $\sqrt{s} \gg m_{c}$.

Now we come to the production of the $X(3872)$. It is necessary to stress that as pointed out in Ref. [15] the production of the $X(3872)$ meson involves many length scales. The creation of the $\bar{c} c$ pair with small relative momentum requires a hard-scattering process with a short-distance scale $m_{c}$. The evolution of the $\bar{c} c$ into a color-singlet hadronic system occurs over a longer length scale $m_{c} v$ or $m_{c} v^{2}$. The evolution of the charmed mesons occurs over an even lower scale $m_{\pi}$. Finally the binding of $D D^{*}$ into the molecular state $X$ occurs over the very long length scale. It is also interesting to notice that the above treatment for the $X(3872)$ production is different with the mixing scheme [16].

Like the case of $Z_{c}$, the leading power operator to produce the $X(3872)$ might have the form:

$$
\langle X|\bar{c} \Gamma c| 0\rangle,\langle X|\bar{q} q| 0\rangle .
$$

For instance for $B_{c} \rightarrow X(3872)$ decays, the $\langle X|\bar{c} \Gamma c| 0\rangle$ contributes at leading power. A consequence is that this contribution is irrespective of the emitted particles in $B_{c}$ decays. Thus the ratios of branching fractions of semileptonic and nonleptonic decays for instance $B_{c} \rightarrow X \ell \bar{v}$ and $B_{c} \rightarrow J / \psi \rho$ can be precisely predicted and tested by data. For more details one can see Ref. [17].

Actually, the leading power behavior has been incorporated in generalized light-cone distribution amplitude (LCDA), where the leading twist LCDA of $p \bar{p}$ and $\pi \pi$ systems is defined by quark fields. For a detailed review see Ref. [18]. The generalized LCDA has recently been used to calculate heavy meson decays in Refs. [19-25] and agreements with relevant data are found.

\section{Prompt $X(3872)$ production at hadron colliders}

The $X(3872)$ is a notable exotic candidate and has received great attention [26]. Its tiny width and the vicinity of its mass to the $D \bar{D}^{*}$ threshold has lead to the speculation as a hadron molecule [27, 28]. A competitive model is compact tetraquark [29], dominated by short-range color interactions. 
The fact that the $X(3872)$ hadron can be copiously produced in hadron collisions has lead to the suspect of the molecular interpretation of the X(3872). Ref. [30] has used the Monte Carlo method and calculated the production rates of $D D^{*}$. By taking a momentum cutoff set by the binding energy, the authors have found the simulated cross section is smaller than data. Actually, this simulation has used the constituent-molecule duality, namely, the production rate of the constituents in the phase space is equivalent to that of the molecule. This is similar with local quark-hadron duality, which often fails for very narrow resonances. To recover the quark-hadron duality, one should include final state interactions which is equivalent to increase the momentum cutoff [31,32]. For the production of ordinary heavy quarkonium, modern EFT approach is nonrelativistic QCD, in which a hadron is nonperturbatively produced by quark fields. Similarly, for hadronic molecules we have established an approach in which the hadron molecule is produced by its constituents, and the low-energy matrix element has been estimated using an effective theory at hadron level in Refs. [33-35]. This approach avoids the use of local constituent-molecule duality, and thus the results should be more reliable.

It has also been argued that "If the $X(3872)$ shares the same molecule nature with light nuclei, one expects similar production cross sections, especially at high values of $p_{\perp}$ " [36]. As we have shown above, unlike the light nuclei like a deutron, the $X(3872)$ contains hidden flavors, and thus the production rate at leading power does not scale according to its low energy nature. This is different with the deutron production, since the deutron has at least six quarks.

\section{Conclusions}

For a reaction involving hadrons, if factorization exists, short-distance and long-distance degrees of freedom decouple from each other. The long-distance physics depends on hadrons' internal structure but does not affect the short-distance coefficients. The short-distance coefficients are calculable using QCD perturbation theory. Using the $e^{+} e^{-} \rightarrow V P$ process, I have analyzed the leading power contribution to the cross section and demonstrated that it is not compatible with the naive constituent scaling rule. This has important consequences in the study of the production of hadron exotics, since the misuse of the constituent scaling rule will sometimes lead to misleading results to the nature of exotic hadrons. Such examples include the production of $Z_{c}^{ \pm}$and $X(3872)$.

\section{Acknowledgement}

I thank the organizers of this workshop, in particular Pietro Colangelo, Fulvia De Fazio, Floriana Giannuzzi, and Stefano Nicotri. I am grateful to Ulf-G. Meißner, Feng-Kun Guo and Qiang Zhao for collaborations. Useful communications with Stanley Brodsky and Richard Lebed are acknowledged. This work is supported in part by the Thousand Talents Plan for Young Professionals, National Natural Science Foundation of China under Grant No.11575110, Natural Science Foundation of Shanghai under Grant No. 15DZ2272100 and No. 15ZR1423100, by the Open Project Program of State Key Laboratory of Theoretical Physics, Institute of Theoretical Physics, Chinese Academy of Sciences, China (No.Y5KF111CJ1), and by Scientific Research Foundation for Returned Overseas Chinese Scholars, State Education Ministry.

\section{References}

[1] H. X. Chen, W. Chen, X. Liu and S. L. Zhu, Phys. Rept. 639, 1 (2016).

[2] G. P. Lepage and S. J. Brodsky, Phys. Rev. D 22, 2157 (1980).

[3] H. Kawamura, S. Kumano and T. Sekihara, Phys. Rev. D 88, 034010 (2013). 
[4] H. Kawamura and S. Kumano, Phys. Rev. D 89, no. 5, 054007 (2014).

[5] S. H. Blitz and R. F. Lebed, Phys. Rev. D 91, no. 9, 094025 (2015).

[6] S. J. Brodsky and R. F. Lebed, Phys. Rev. D 91, 114025 (2015).

[7] W. C. Chang, S. Kumano and T. Sekihara, Phys. Rev. D 93, no. 3, 034006 (2016).

[8] F. K. Guo, U. G. Meißner and W. Wang, arXiv:1607.04020 [hep-ph].

[9] C. W. Bauer, S. Fleming and M. E. Luke, Phys. Rev. D 63, 014006 (2000).

[10] C. W. Bauer, S. Fleming, D. Pirjol and I. W. Stewart, Phys. Rev. D 63, 114020 (2001).

[11] T. Becher, A. Broggio and A. Ferroglia, Lect. Notes Phys. 896 (2015).

[12] C. D. Lu, W. Wang and Y. M. Wang, Phys. Rev. D 75, 094020 (2007).

[13] M. Ablikim et al. [BESIII Collaboration], Phys. Rev. Lett. 110, 252001 (2013).

[14] Z. Q. Liu et al. [Belle Collaboration], Phys. Rev. Lett. 110, 252002 (2013).

[15] E. Braaten, Phys. Rev. D 73, 011501 (2006).

[16] C. Meng, H. Han and K. T. Chao, arXiv:1304.6710 [hep-ph].

[17] W. Wang and Q. Zhao, Phys. Lett. B 755, 261 (2016).

[18] M. Diehl, Phys. Rept. 388, 41 (2003).

[19] C. H. Chen and H. n. Li, Phys. Lett. B 561, 258 (2003).

[20] U. G. Meißner and W. Wang, Phys. Lett. B 730, 336 (2014).

[21] W. F. Wang, H. C. Hu, H. n. Li and C. D. Lü, Phys. Rev. D 89, no. 7, 074031 (2014).

[22] W. Wang and R. L. Zhu, Phys. Lett. B 743, 467 (2015).

[23] W. F. Wang, H. n. Li, W. Wang and C. D. Lü, Phys. Rev. D 91, no. 9, 094024 (2015).

[24] Y. J. Shi and W. Wang, Phys. Rev. D 92, no. 7, 074038 (2015).

[25] C. Hambrock and A. Khodjamirian, Nucl. Phys. B 905, 373 (2016).

[26] S. K. Choi et al. [Belle Collaboration], Phys. Rev. Lett. 91, 262001 (2003).

[27] N. A. Tornqvist, Phys. Lett. B 590, 209 (2004).

[28] C. Hanhart, Y. S. Kalashnikova, A. E. Kudryavtsev and A. V. Nefediev, Phys. Rev. D 76, 034007 (2007).

[29] L. Maiani, F. Piccinini, A. D. Polosa and V. Riquer, Phys. Rev. D 71, 014028 (2005).

[30] C. Bignamini, B. Grinstein, F. Piccinini, A. D. Polosa and C. Sabelli, Phys. Rev. Lett. 103, 162001 (2009).

[31] P. Artoisenet and E. Braaten, Phys. Rev. D 81, 114018 (2010).

[32] P. Artoisenet and E. Braaten, Phys. Rev. D 83, 014019 (2011).

[33] F. K. Guo, U. G. Meißner and W. Wang, Commun. Theor. Phys. 61, 354 (2014).

[34] F. K. Guo, U. G. Meißner, W. Wang and Z. Yang, Eur. Phys. J. C 74, no. 9, 3063 (2014).

[35] F. K. Guo, U. G. Meißner, W. Wang and Z. Yang, JHEP 1405, 138 (2014).

[36] A. Esposito, A. L. Guerrieri, L. Maiani, F. Piccinini, A. Pilloni, A. D. Polosa and V. Riquer,

Phys. Rev. D 92, no. 3, 034028 (2015). 